\title{
Control Strategy Research of High-frequency Position Response Servo System for Electromechanical Actuator
}

\author{
Zaiping Zheng ${ }^{1, a}$, Yi Wang ${ }^{1, b}$, Yuping Huang ${ }^{1, c}$, Hongxing $\mathrm{Wu}^{2, \mathrm{~d}}$ \\ ${ }^{1}$ Beijing Research Institute of Precise Mechatronic Controls, Beijing, 100048, China \\ ${ }^{2}$ Department of Electrical Engineering, Harbin Institute of Technology, Harbin, 150001, China \\ aalan98422@sina.com, bjacluwu@163.com, cshanxi247@163.com, dwhx0422@sina.com
}

Keywords: servo system, slide mode variable structure, position servo

\begin{abstract}
This paper presents a method of improving the position response speed of the servo system based on electromechanical actuator position servo system. In this paper, a novel decouple technique is derived from PMSM decouple state equation, separating direct axis current from quadrature axis current ulteriorly, and the impact of EMF in high dynamic conditions is overcome. Based on analysing the principle of slide mode variable structure (SMVS), the boundary conditions of SMVS control and equivalent control strategy, the method of applying SMVS in the servo speed system has been put up, and the SMVS controller for the speed-loop has been built. The system uses feed-forward control to compensate the position deviation of PMSM in steering engine. Feed-forward transfer function is analysed and feed-forward controller is established to ensure the performance of electromechanical actuator applied in missile.
\end{abstract}

\section{Introduction}

Electromechanical actuator position servo system has advantages of small volume, light weight, small time constant and excellent load property and so on, and more and more military experts pay attention to it[1]. It will be developing tendency that electromechanical actuator takes place of traditional hydraumatic or pneumatic actuator in the future actuator control field. DC motor has good speed control features, but the armature and commutator are easy to get oxidation corrosion, so that the reliability is bad, which has restrict electromechanical actuator's development.

Permanent magnet synchronous motor(PMSM) with vector control has advantages of fast dynamic response, high precision in steady-state, low torque ripple and smooth operation characters in low speed, meanwhile the speed range is wide[2]. In recent years, with the growing of microprocessor technique and control theory, combining classic control theory with modern control theory, applying it to high precision, fast response AC servo system has become a significant research. Nowadays, control theory applied to AC servo system mainly contains: classic PID control, fuzzy control [3], SMVS control[4][5][6], self adaptive control and so on.

The purpose of this paper is to overcome hydraumatic and pneumatic actuator's shortage, ameliorate the dynamic frequency response character of actuator system. Electromechanical actuator based on PMSM servo system can accomplish complicated servo task. In this paper, the authors have done deep research about position feed-forward method and SMVS method, aiming at improving position servo system's response speed, enhancing its robustness and anti-interference performance. Position feed-forward controller is designed in position loop and SMVS controller is designed in speed-loop. The simulation and test results show the PMSM servo system has higher dynamic character and it can satisfy the target of actuator system.

\section{PMSM Mathematical Model and Decouping}

The mathematic model of PMSM in d-q axis can be derived from CLARK transform and PARK transform as follows[7][8]: 


$$
\left\{\begin{array}{l}
u_{d}=R_{a} i_{d}+\frac{d \psi_{d}}{d t}-\psi_{q} P_{m} \omega_{m} \\
u_{q}=R_{a} i_{q}+\frac{d \psi_{q}}{d t}+\psi_{d} P_{m} \omega_{m} \\
T_{e}=\frac{3}{2} P_{m}\left(\psi_{d} i_{q}-\psi_{q} i_{d}\right)
\end{array}\right.
$$

In the type, $u_{d}$ and $u_{q}$ are d-axis voltage and q-axis voltage after transforming; $i_{d}$ and $i_{q}$ are d-axis current and q-axis current after transforming; $R_{a}$ is winding resistance; $\omega_{m}$ is mechanical angular velocity; $P_{m}$ is polar pairs; $\psi_{d}$ and $\psi_{q}$ are flux linkage in d-axis and q-axis.

If the saturation of magnetic circuit is ignored, the equation will be:

$$
\left\{\begin{array}{l}
\psi_{d}=L_{d} i_{d}+\psi_{f m} \\
\psi_{q}=L_{q} i_{q}
\end{array}\right.
$$

In the type, $L_{d}$ and $L_{q}$ is d-q axis inductance; $\psi_{f m}$ is amplitude of permanent magnet flux coupling with stator winding flux.

From (1) and (2) we can get PMSM's model as follows :

$$
\left\{\begin{array}{l}
u_{d}=R_{a} i_{d}+L_{d} \frac{d i_{d}}{d t}-P_{m} \omega_{m} L_{q} i_{q} \\
u_{q}=R_{a} i_{q}+L_{q} \frac{d i_{q}}{d t}+P_{m} \omega_{m} L_{d} i_{d}+P_{m} \omega_{m} \psi_{f} \\
T_{e}=\frac{3}{2} P_{m}\left[\psi_{f} i_{q}+\left(L_{d}-L_{q}\right) i_{d} i_{q}\right]
\end{array}\right.
$$

PMSM mechanical motion equation is as follow :

$$
T_{e}=J \frac{d \omega_{m}}{d t}+T_{l}+B \omega_{m}
$$

In the type, $T_{l}$ is load torque; $J$ is inertia; $B$ is friction coefficient.

From this equation we can see PMSM block diagram in Fig.1 :

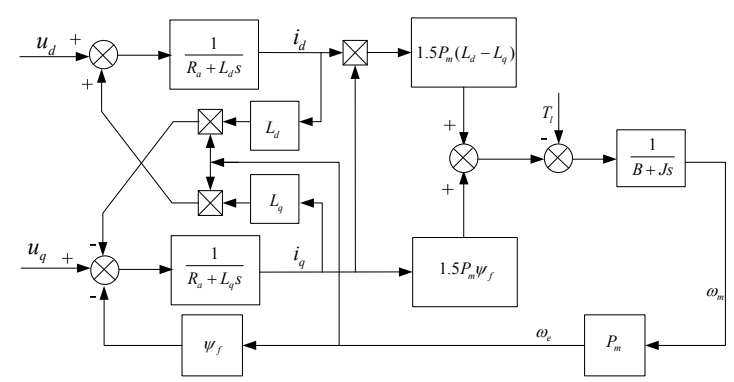

Fig.1. Block diagram of PMSM mathematic model

Usually, PMSM used in servo system has the structure of surface mounted magnet which make $\mathrm{d}$-axis inductance equals to q-axis inductance, that is $L_{d}=L_{q}=L$. So PMSM model can simplify as follows :

$$
\left\{\begin{array}{l}
u_{d}=R_{a} i_{d}+L \frac{d i_{d}}{d t}-P_{m} \omega_{m} L i_{q} \\
u_{q}=R_{a} i_{q}+L \frac{d i_{q}}{d t}+P_{m} \omega_{m} L i_{d}+P_{m} \omega_{m} \psi_{f} \\
T_{e}=\frac{3}{2} P_{m} \psi_{f} i_{q}
\end{array}\right.
$$

From(5) state equation of PMSM is written as follows :

$$
\left[\begin{array}{l}
\frac{d i_{d}}{d t} \\
\frac{d i_{q}}{d t} \\
\frac{d \omega_{m}}{d t}
\end{array}\right]=\left[\begin{array}{ccc}
-\frac{R_{a}}{L} & P_{m} \omega_{m} & 0 \\
-P_{m} \omega_{m} & -\frac{R_{a}}{L} & -\frac{P_{m} \psi_{f}}{L} \\
0 & \frac{3 P_{m} \psi_{f}}{2 J} & -\frac{B \omega_{m}}{J}
\end{array}\right]\left[\begin{array}{l}
i_{d} \\
i_{q} \\
\omega_{m}
\end{array}\right]+\left[\begin{array}{c}
\frac{u_{d}}{L} \\
\frac{u_{q}}{L} \\
-\frac{T_{l}}{J}
\end{array}\right]
$$

We can see from above that after simplifying the mathmatic model is a second order, non-linear, 
multivariable equation.

\section{Controller Design of Speed_loop and Position_loop}

Often PMSM servo system can be simplified as a second order system, so in this section the controller design of SMVS in second order system is introduced. And then the parameters in real syetem are considered to ameliorate speed-loop performance. SMVS controller is employed. According to PMSM syetem feature switching function and control law are designed to enhance the robustness of PMSM servo system.

Variable structure control is a high-speed switch feed-back control method. The fundemental difference between this method and the other methods is the control law and syetem structure is discontinuous on slide mode surface, its structure is not fixed but varying in the transient progress [9][10].

Second Order SMVS Controller Assume the state space equation of classic second order system is:

$$
\left\{\begin{array}{l}
\dot{x}_{1}=x_{2} \\
\dot{x}_{2}=-a_{1} x_{1}-a_{2} x_{2}-b u+f
\end{array}\right.
$$

Where $x_{1}, x_{2}$ are state variables, $a_{1}, a_{2}$ and $b$ are constants or time varying parameters, $u$ is output and $f$ is interference. Considering the in real system the variation range is limited, the range is as follows:

$$
\left\{\begin{array}{l}
a_{1 \min } \leq a_{1} \leq a_{1 \max } \\
a_{2 \min } \leq a_{2} \leq a_{2 \max } \\
b_{\min } \leq b \leq b_{\max }
\end{array}\right.
$$

In SMVS control, switching function is $s(x)$, control function is $u(x)$, as follows:

$$
u(x)= \begin{cases}u^{+}(x) & s(x)>0 \\ u^{-}(x) & s(x)<0\end{cases}
$$

Where $u^{+}(x) \neq u^{-}(x)$ and $c>0$.

For $s(x)=c x_{1}+x_{2}$, to ensure accessibility of sliding mode dynamics, it requires:

From Fig. 2 we can see system motion trail, where $s(x)=c x_{1}+x_{2}=0$ is slide mode surface. When the trail moves into slide mode surface, it begins to slide toward neighborhood with switching motion in high-speed, finally it tends to be $: \dot{s}=0$ and $s=0$. The equation $s(x)=c x_{1}+x_{2}=0$ is required, and the solution is:

$$
\left\{\begin{array}{l}
x_{1}=x_{1}(0) e^{-c t} \\
x_{2}=-c x_{1}(0) e^{-c t}
\end{array}\right.
$$

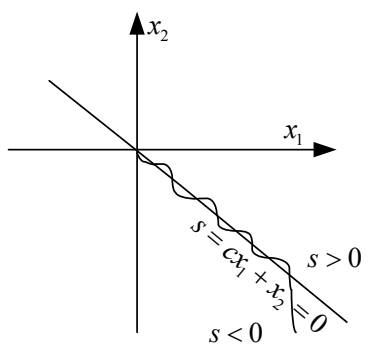

Fig.2. State trajectory of second order slide mode system

To a certain second order system, when designing switching function and the parameters of control function, the accessibility of slide mode motion should be guaranteed, that is: 
$s \dot{s}=s\left(c \dot{x}_{1}+\dot{x}_{2}\right)<0$

By (7) and (12) we have:

$s\left(c x_{2}-a_{1} x_{1}-a_{2} x_{2}-b u+f\right)<0$

Here control function $u(x)$ is:

$$
u(x)=\varphi_{1} x_{1}+\varphi_{2} x_{2}
$$

And let:

$$
\varphi_{1}=\left\{\begin{array}{ll}
\alpha_{1}, & x_{1} s>0 \\
\beta_{1}, & x_{1} s<0
\end{array}, \varphi_{2}= \begin{cases}\alpha_{2}, & x_{2} s>0 \\
\beta_{2}, & x_{2} s<0\end{cases}\right.
$$

By (13) and (14) we have:

$$
-\left(a_{1}+b \varphi_{1}\right) x_{1} s+\left(c-a_{2}-b \varphi_{2}\right) x_{2} s+f s<0
$$

Ignoring $\mathrm{f}$, the following four conditions are discussed:

$$
\text { a. }\left\{\begin{array} { l } 
{ x _ { 1 } s > 0 } \\
{ x _ { 2 } s > 0 }
\end{array} \text { b. } \left\{\begin{array} { l } 
{ x _ { 1 } s > 0 } \\
{ x _ { 2 } s < 0 }
\end{array} \text { c. } \left\{\begin{array} { l } 
{ x _ { 1 } s < 0 } \\
{ x _ { 2 } s > 0 }
\end{array} \text { d. } \left\{\begin{array}{l}
x_{1} s<0 \\
x_{2} s<0
\end{array}\right.\right.\right.\right.
$$

Then we have:

$$
\left\{\begin{array} { l } 
{ \alpha _ { 1 } > - \frac { a _ { 1 \operatorname { m i n } } } { b _ { \operatorname { m a x } } } } \\
{ \beta _ { 1 } < - \frac { a _ { 1 \operatorname { m i n } } } { b _ { \operatorname { m a x } } } }
\end{array} \quad \left\{\begin{array}{l}
\alpha_{2}>\frac{c-a_{2 \min }}{b_{\max }} \\
\beta_{2}<\frac{c-a_{2 \max }}{b_{\max }}
\end{array}\right.\right.
$$

SMVS Control Design In Speed-loop System speed controller is designed using SMVS in second order system dedign method which is mentioned before. Zero state variable are $x_{1}=\omega^{*}-\omega$, $x_{2}=x_{1}^{*}=-d \omega / d t=-\dot{\omega}_{m}$, for output is $u=\dot{i}_{q}^{*}$, ignoring viscosity factor $B$, and PMSM system's state equations based on rotor flux field-oriented vector control method will be:

$$
\left\{\begin{array}{l}
\dot{x_{1}}=x_{2} \\
\dot{x_{2}}=-\frac{3 P_{m} \psi_{f} \omega_{m}}{2} \dot{i_{q}}
\end{array}\right.
$$

For switching function $s(x)=c x_{1}+x_{2}$, where $c$ is constant, control function $u=\varphi_{1} x_{1}+\varphi_{2} x_{2}$, the space state equations(19) and controller design method from(18) shows:

$$
\left\{\begin{array} { l } 
{ \alpha _ { 1 } > 0 } \\
{ \beta _ { 1 } < 0 }
\end{array} \quad \left\{\begin{array}{l}
\alpha_{2}>-\frac{2 c J}{3 P_{m} \varphi_{f}} \\
\beta_{2}<-\frac{2 c J}{3 P_{m} \varphi_{f}}
\end{array}\right.\right.
$$

Design of Position Feed-foaward Controller When $\mathrm{P}$ (proportion) controller is used in position loop, as to prevent position overshoot, usually proportional gain can't be too large, so quick response of the whole system is limited. To get higher location precision, compensate position error and enhance controller performance, PI controller is introduced. Though to some extent the position precision is improved, it is still far from fast response requirement. In paper[11] a feed-back controller with robustness based on interference observer is designed; a feed-forward controller is used so that position response speed is raised.

Generally a feed-forward controller can restrain interference to system position and enhance the whole system's robustness. In this paper, SMVS servo system is mainly discussed, controller in position loop is improved; feed-forward controller is added; Parameters in feed-forward controller is designed to adapt real system. Experiment results show position response speed is obviously raised when feed-forward controller is used in position servo system.

No matter single-loop or multi-loop servo system, feed-back control achieves by Automatic Position Reporting (APR). The change of reference signal works via APR. To guarantee system's stability, signal track speed shouldn't be too fast when design APR. If we want to make further 
improvement on position track performance, feed-forward control from reference signal along with feed-back control consists of complex control system, the block diagram is shown in Fig.3:

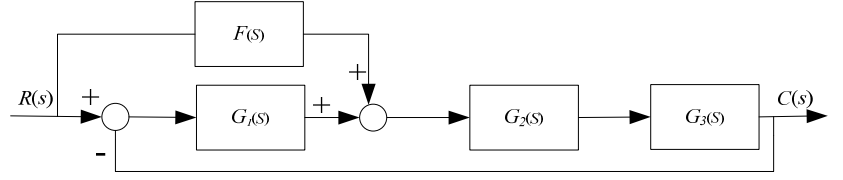

Fig.3. Block diagram of system transfer function with position feed-back controller

From block above we will get the close-loop transfer function is:

$G(s)=\frac{C(s)}{R(s)}=\frac{F(s) G_{2}(s) G_{1}(s)+G_{1}(s) G_{2}(s) G_{3}(s)}{1+G_{1}(s) G_{2}(s) G_{3}(s)}$

From the close-loop transfer function we can see, when $F(s) G 1(s) G 2(s)=1$, that is $F(s)=1 / G 1(s) G 2(s), G(s)=1$. That means, no matter the input is ramp signal or acceleration signal, system output will repeat input completely, so fast response, no-overshoot and high precision is ensured. The feed-forward controller's transfer function is as follows:

$$
F(s)=\frac{T_{v} s^{2}+s}{K_{v}}
$$

Feed-forward controller's transfer function $F(s)$ can be seen as acceleration feed-forward $T_{v} s^{2} / K_{v}$, which makes both speed signal and acceleration signal have feed-forward effect together with speed feed-forward $\mathrm{s} / K_{v}$. From system open-loop character it can be known this method can improve system response speed to position signal apparently. Close-loop control eliminates overshoot in system and control requirement is guaranteed.

Because that the real motor servo control system uses discrete control strategy, the parameters in acceleration feed-forward and speed feed-forward, or there will be oscillation in the system.

\section{Generator Performance Analysis}

Firstly the reference position signal (sinusoidal wave with $\pm 110^{\circ}$ amplitude) is given, feed-forward controller is used in position-loop and in speed-loop SMVS controller is used, and the simulation results are as follows. Fig. 4 -Fig.6 show given and actual signal in $5 \mathrm{~Hz}, 10 \mathrm{~Hz}$ and $15 \mathrm{~Hz}$ of system position.

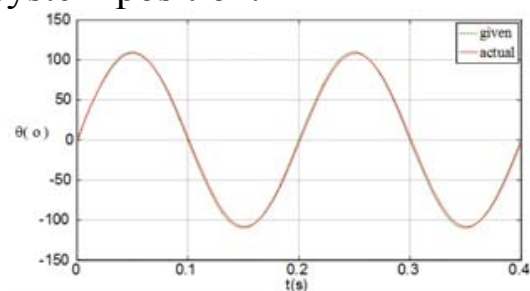

Fig.4.position response in $5 \mathrm{~Hz}$

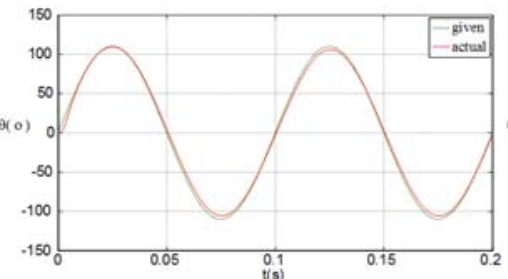

Fig.5.position response in $10 \mathrm{~Hz}$

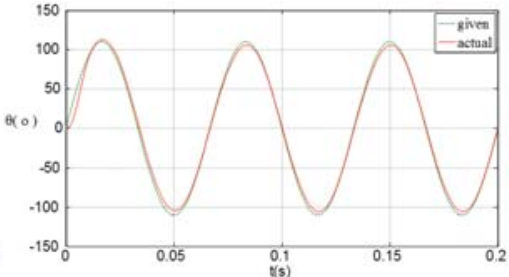

Fig.6.position response in $15 \mathrm{~Hz}$

We can see from the table below, in no-load condition, compared with PI control, SMVS control has faster response speed and higher steady-state precision.

Table.1. Statistical table of the frequency-phase and frequency-amplitude of response curve

\begin{tabular}{c|c|c|c|c}
\hline \multirow{2}{*}{$\begin{array}{c}\text { Freq } \\
(\mathrm{Hz})\end{array}$} & \multicolumn{2}{|c|}{ Phase shifting $\left(^{\circ}\right)$} & \multicolumn{2}{c}{ Amplitude ratio(\%) } \\
\cline { 2 - 5 } & PI & $\begin{array}{c}\text { VSC and } \\
\text { feed-forward }\end{array}$ & PI & $\begin{array}{c}\text { VSC and } \\
\text { feed-forward }\end{array}$ \\
\hline 1 & 1.94 & 1.84 & 99.9 & 99.9 \\
\hline 5 & 9.54 & 9 & 98.7 & 98.9 \\
\hline 10 & 18.36 & 17.64 & 94.8 & 95.84 \\
\hline 15 & 27.07 & 25.9 & 89.3 & 91.3 \\
\hline 18 & 38.88 & 31.72 & 87.3 & 87.01 \\
\hline
\end{tabular}

Armature current waveform is shown in Fig.7 when reference sine-wave's amplitude is $110^{\circ}$ in $15 \mathrm{~Hz}$. 

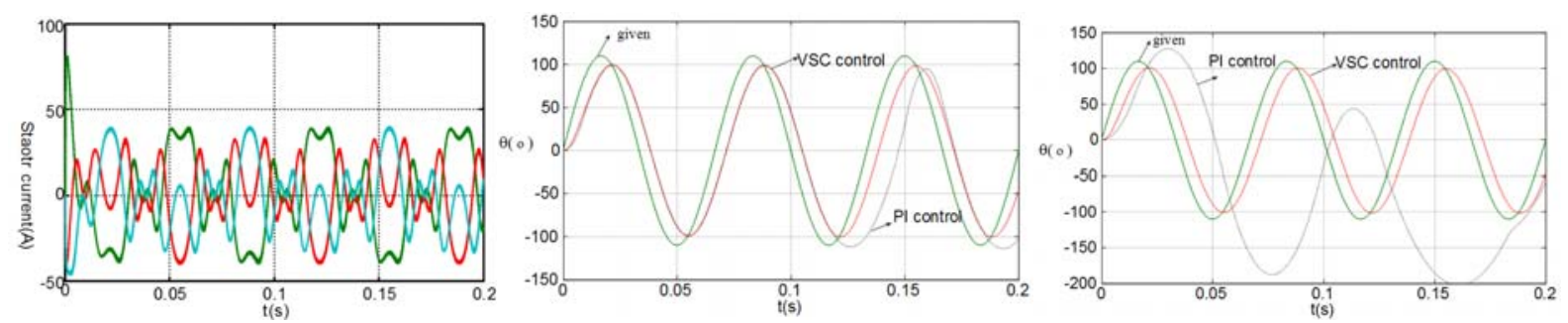

Fig.7. Stator current waveform Fig.8. position response under sudden $5 \mathrm{Nm}$ load Fig.9. position response under varying inertia

SMVS control' shortage is, stator current may be large enough to have system protection work in when starting, and in real system design it should be considered.

Reference sine-wave's amplitude is $110^{\circ}$ in $15 \mathrm{~Hz}$. Simulation results are compared when the parameters and load of the motor have changed.

In $0 \mathrm{~s}$ the motor has no load and in $0.1 \mathrm{~s}$ torque step signal $(5 \mathrm{~N} . \mathrm{m})$ is loaded in motor.

From Fig. 8 we can see, when torque step signal is suddenly loaded, position response performance becomes bad in PI control while in VSC control position response curve changes little by torque interference.

Simulation result that inertia changes from $5.7 \times 10^{-4} \mathrm{~N} . \mathrm{m}^{2}$ (no load) to $1.14 \times 10^{-3} \mathrm{~N} . \mathrm{m}^{2}$ is shown as follows:

As shown in Fig. 9, when inertia changes, position response performance in PI control is poor and even position response can't track the reference signal; while SMVS control makes good position response. SMVS controller isn't sensitive to varying parameters and interference, and has strong robustness.

Reference square wave's amplitude is $110 \mathrm{o}$ in $1 \mathrm{~Hz}$, simulation result is shown in Fig.10:

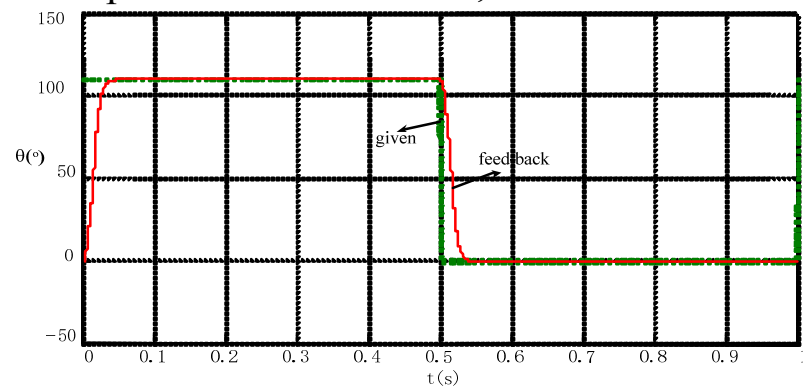

Fig.10.waveform of square wave in $1 \mathrm{~Hz}$, amplitude $110 \mathrm{o}$

Comparing the simulation results of PI control with SMVS control in speed-loop controller design, we can know that SMVS control is not sensitive to varying parameters and have strong anti-interference ability. SMVS controller in speed-loop and feed-forward controller in position-loop improve robustness of PMSM position servo system efficiently, and system dynamic response ability gets enhanced.

\section{Conclusion}

In this paper, PMSM position servo system is studied and current-loop, speed-loop and position-loop in servo system in is analysed and designed based on vector control theory. SMVS controller in speed-loop is designed aiming at electromechanical actuator position servo system. Feed-forward controller is added in to guarantee position precision and no-overshoot and to accelerate position tracking speed. Simulation results denote this strategy can ensure high-precision, rapidity and no-overshoot. Besides, this method has some advantages like strong anti-interference ability, no-sensitive to interference and robustness. The rationality and effectiveness of modified control theory get proved.

\section{Acknowledgement}

In this paper, the research was sponsored by Natural Science Foundation of China (Project No. 51177025) and China Postdoctoral Science Foundation Funded Project. 


\section{References}

[1] Huan Yuping; Wu Hongxing; Kou Baoquan; Li Liyi; Research on rotating encoder zero-position location based on missile steering gear. 3rd International Conference on Sensing Technology, Tainan: [s.n.], 2008:269-272.

[2] Wu Hongxing; Li Liyi; Kou Baoquan; Zheng Ping; The research on energy regeneration of permanent magnet synchronous motor used of hybrid electric vehicle. IEEE Vehicle Power and Propulsion Conference, Harbin: Harbin Institute of Technology Publishers, 2008:206-208

[3] Liyi Li; Junjie Hong; Hongxing Wu; Baoquan Kou; Peng Li; Qiguo Li; Section crossing drive with fuzzy-PI controller for the long stroke electromagnetic launcher. 14th Symposium on Electromagnetic Launch Technology, Victoria:[s.n.],2009:363-367

[4] Papnpen, K.; Konghirun, M. An Improved Sliding Mode Observer for Speed Sensorless Vector Control Drive of PMSM. Power Electronics and Motion Control Conference, 2006. IPEMC '06. CES/IEEE 5th International 2006(2):1-5

[5] O. Barambones*; A. J. Garrido; F. J. Maseda; P. Alkorta; An adaptive Sliding Mode Control Law for Induction Motors Using Field Oriented Control Theory. Proceedings of the 2006 IEEE International Conference on Control Applications, 2006:1008-1013

[6] Slotine. J. J; Sastry. S. S; Tracking control of nonlinear systems using sliding surfaces with application to robot manipulator. International Journal of Control, 1983, 38(2):465-492

[7] Ying YAN; Jiangguo Zhu; Simulation of a direct torque controlled PMSM drive incorporating structural and Saturation saliencies. Power Electronics Specialists Conference. 2006. PESC 37th IEEE. 2006:1-6

[8] Wu Hong-xing; Hong Jun-jie; Li Li-yi; Research for the control system of a pulsed power permanent linear synchronous motor. 14th Symposium on Electromagnetic Launch Technology, Victoria:[s.n.], 2008: 307-310

[9] Utkin V I.; Variable structure systems with sliding modes. IEEE Transactions Automatic Control, 1977, 22(2): 212-222

[10] Kawamura. A. Itoh. H; Sakamoto K.; Chattering reduction of disturbance observer based sliding mode control. IEEE Transactions on Industry Applications, 1994, 30(2): 456-461

[11] Ho Seong Lee; Masayashi Tomizuka; Robust Motion Controller Design for High-Accuracy Positioning Systems. IEEE Transactions on industry electronics. 1996, 43(1): 48-55 